\title{
BOHR AND ROGOSINSKI ABSCISSAS FOR ORDINARY DIRICHLET SERIES.
}

\author{
LEV AIZENBERG, VICTOR GOTLIB, ALEKOS VIDRAS
}

\begin{abstract}
We prove that the abscissas of Bohr and Rogosinski for ordinary Dirichlet series, mapping the right half-plane into the bounded convex domain $G \subset \mathbb{C}$ are independent of the domain $G$. Furthermore, we obtain new estimates about these abscissas.
\end{abstract}

\section{Preliminaries}

Let us recall the theorem of H.Bohr [19] in 1914.

Theorem 1.1. If a power series

$$
f\left(z_{1}\right)=\sum_{k=0}^{\infty} c_{k} z_{1}^{k}
$$

converges in the unit disk $U_{1}$ and its sum has modulus less than 1 , then

$$
\sum_{k=0}^{\infty}\left|c_{k} z_{1}^{k}\right|<1,
$$

if $\left|z_{1}\right|<\frac{1}{3}$. Moreover, the constant $\frac{1}{3}$ cannot be improved.

For reasons of convenience we write the inequality (1.2) in the following equivalent form

$$
\sum_{k=1}^{\infty}\left|c_{k} z_{1}^{k}\right|<1-\left|c_{0}\right| .
$$

Generalizations and modifications of this result can be found in [2], [4, 5] - 17, [1], 14, 24, 25, 27, 30, 31]. On the other hand, formulation of Bohr theorem in several complex variables appeared very recently. Given a complete Reinhardt domain $\mathcal{D} \subset \mathbb{C}^{n}$, we denote by $R(\mathcal{D})$ the largest non-negative number $r$ with the property that if the power series

$$
f(z)=\sum_{|\alpha| \geq 0} c_{\alpha} z^{\alpha}, z \in \mathcal{D},
$$

AMS classification number: 30B50, 30A10.

Keywords: Isometric Bohr abscissa, Rogosinski abscissa, Dirichlet series. 
where as usual, $\alpha=\left(\alpha_{1}, \ldots, \alpha_{n}\right) \in \mathbb{N}_{0}^{n}, z^{\alpha}=z_{1}^{\alpha_{1}} \ldots z_{n}^{\alpha_{n}},|\alpha|=\alpha_{1}+$ $\ldots \alpha_{n}$, converges in $\mathcal{D}$ and the modulus of its sum is less than 1 , then

$$
\sum_{|\alpha| \geq 1}\left|c_{\alpha} z^{\alpha}\right|<1-\left|c_{0}\right|
$$

in the homothetic domain $\mathcal{D}_{r}=r \mathcal{D}$. Here $c_{0}=c_{0, \ldots, 0}$. A variety of results, related to this particular $R(\mathcal{D})$ or other multidimensional generalizations of Bohr radius are found in [1] - [11], [14] - [16], 20] [24]. Key results, used by us in the present paper, were obtained in [4], [8], [12].

Let $\widetilde{G}$ be a convex hull of the domain $G \subset \mathbb{C}$. For a a complete Reinhardt domain $\mathcal{D}$ in $\mathbb{C}^{n}$, denote by $R(\mathcal{D}, G)$ the largest $r \geq 0$ such that if the function $f(z)$ from (1.3) is holomorphic in $\mathcal{D}, f(\mathcal{D}) \subset G$ and $\widetilde{G} \neq \mathbb{C}$, then

$$
\sum_{|\alpha| \geq 1}\left|c_{\alpha} z^{\alpha}\right|<\operatorname{dist}\left(c_{0}, \partial \widetilde{G}\right)
$$

in homothety $\mathcal{D}_{r}$. A point $p \in \partial G$ is called a point of convexity if $p \in \partial \widetilde{G}$. A point of convexity $p$ is called regular if there exists a disk $U \subset G$ so that $p \in \partial U$.

The following result is a consequence of a more general result, proved [4]. We state it in a suitable for us form for the purposes of the present article.

Theorem 1.2. If $\widetilde{G} \neq \mathbb{C}$, then $R(\mathcal{D}, G)$ is not smaller than $R\left(\mathcal{D}, U_{1}\right)$. If $\partial G$ contains at least one point of regular convexity, then

$$
R(\mathcal{D}, G)=R\left(\mathcal{D}, U_{1}\right) .
$$

Corollary 1.1. If the domain $G$ is convex and $G \neq \mathbb{C}$, then $R(\mathcal{D}, G)$ is independent of the choice of $G$.

Besides the Bohr radius we will use the radius of Rogosinski, whose classic result of 1923, [28, 29, 26], is described in the following statement:

Theorem 1.3. If the function $f\left(z_{1}\right)$ from (1.1) is holomorphic in the unit disk $U_{1}$ and $\left|f\left(z_{1}\right)\right|<1$ in $U_{1}$, then all of its partial sums are less than 1 in the disk of radius $\frac{1}{2}$, that is:

$$
\left|\sum_{k=0}^{m} c_{k} z_{1}^{k}\right|<1
$$

for $\left|z_{1}\right|<\frac{1}{2}$ and this radius is sharp. 
The following result is the consequence of more general fact proved in [8]. Again, we are going to use its particular, convenient for us, formulation: let $\mathcal{A}$ be a lattice in $\mathbb{N}_{0}^{n}$, which is represented by

$$
\mathcal{A}=\left\{\alpha \in \mathbb{N}_{0}^{n}: m_{1} \alpha_{1}+\cdots+m_{n} \alpha_{n} \leq m\right\},
$$

where all the numbers $m_{1}, \ldots, m_{n}, m$ belong to $\mathbb{N}_{0}$ and have no common divisor.

Theorem 1.4. Let the function $f(z)$ in (1.3) be holomorphic in the Reinhardt domain $\mathcal{D}$ and $f(\mathcal{D}) \subset G$, where $G$ is a convex domain in $\mathbb{C}$ so that $G \neq \mathbb{C}$. Then the polynomial (partial sum)

$$
\sum_{\alpha \in \mathcal{A}} c_{\alpha} z^{\alpha}
$$

maps $\mathcal{D}_{\mathcal{A}}$ into $G$, where

$$
\mathcal{D}_{\mathcal{A}}=\left\{z \in \mathbb{C}^{n}:\left(\frac{z_{1}}{r_{m}^{m_{1}}}, \ldots, \frac{z_{n}}{r_{m}^{m_{n}}}\right) \in \mathcal{D}\right\}
$$

does not depend on $G, r_{1}=\frac{1}{2}, r_{2}=\frac{\sqrt{3}}{8}$ and for $l \geq 3$ the number $r_{l}$ is the unique positive solution of the equation

$$
1-r-2 r^{l+1}=0 .
$$

In the present article we investigate the Dirichlet series

$$
f(s)=\sum_{n=1}^{\infty} \frac{a_{n}}{n^{s}},
$$

converging in the right half-plane $\Pi=\{s \in \mathbb{C}: \Re s=\sigma>0\}$ and $f(s) \in H^{\infty}(\Pi)$, that is $\|f\|=\sup _{s \in \Pi}|f(s)|<\infty$. Following [12], we call isometric Bohr abscissa $\mathbf{b}$ the non-negative real number defined as the infimum of those $\sigma \geq 0$ such that for all $f \in H^{\infty}(\Pi)$ which can be expressed as a Dirichlet series (1.4), the following holds

$$
\sum_{n=1}^{\infty} \frac{\left|a_{n}\right|}{n^{\sigma}}<\|f\| .
$$

For reasons of convenience, we write (1.5) as

$$
\sum_{n=2}^{\infty} \frac{\left|a_{n}\right|}{n^{\sigma}} \leq\|f\|-a_{1} .
$$

The next result was obtained in [12]. 
Theorem 1.5. For the isometric Bohr abscissa the following estimates are valid

$$
1.5850 \cdots=\frac{\log 3}{\log 2} \leq \mathbf{b}<1.8154
$$

If $f \in H^{\infty}(\Pi)$ and $\|f\|=1$, then

$$
\left|a_{1}\right|^{2}+\sum_{n=2}^{\infty} \frac{\left|a_{n}\right|}{n^{\sigma}} \leq 1
$$

where $\sigma<1.7287$.

Analogously, to the Bohr radius $R(\mathcal{D}, G)$, the isometric Bohr abscissa $\mathbf{b}(G)$, where $G$ is a domain in $\mathbb{C}, \widetilde{G} \neq \mathbb{C}$, is defined as the infimum of those $\sigma \geq 0$ such that if $f \in H^{\infty}(\Pi), f(s)$ is like in (1.4) and $f(\Pi) \subset G$, then

$$
\sum_{n=2}^{\infty} \frac{\left|a_{n}\right|}{n^{\sigma}}<\operatorname{dist}\left(a_{1}, \partial \widetilde{G}\right) .
$$

Furthermore, we define the Rogosinski abscissa $\mathbf{r}(G)$ for functions $f \in$ $H^{\infty}(\Pi), f(\Pi) \subset G$, as the infimum of $\sigma^{\prime} \geq 0$, such that for all partial sums of the series (1.4)

$$
\sum_{n=1}^{k} \frac{a_{n}}{n^{s}}=P_{k}(s)
$$

the inclusion $P_{k}\left(\Pi_{\sigma^{\prime}}\right) \subset G$ is valid, where $\Pi_{\sigma^{\prime}}=\left\{s \in \mathbb{C}: \sigma>\sigma^{\prime}\right\}$.

\section{The MAIN RESUlTS}

Theorem 2.1. Let $G$ be a be a bounded domain in $\mathbb{C}$. Then the isometric Bohr abscissa $\mathbf{b}(G)$ is not larger then the isometric Bohr abscissa $\mathbf{b}\left(U_{1}\right)$. If $\partial G$ contains at least one regular point of convexity, then $\mathbf{b}(G)=\mathbf{b}\left(U_{1}\right)$.

Proof: We are going to exploit the connection between classical Dirichlet series and the power series in the infinite dimensional polydisc found by H.Bohr in [18]. Consider the series (1.4). Every natural number $n$ is product of its prime factors

$$
n=p_{1}^{\alpha_{1}} \ldots p_{m}^{\alpha_{m}} .
$$

We set $z=\left(p_{1}^{-s}, p_{2}^{-s}, \ldots\right)$. Then

$$
f(s)=\sum_{n=1}^{\infty} a_{n}\left(p_{1}^{-s}\right)^{\alpha_{1}} \ldots\left(p_{m}^{-s}\right)^{\alpha_{m}}=\sum_{n=1}^{\infty} a_{n} z_{1}^{\alpha_{1}} \ldots z_{m}^{\alpha_{m}}
$$


where $m=m(n)$. If the power series (1.4) converges absolutely for $\sigma=\sigma_{0}$, then the set of values of $f(s)$ on the vertical line $\{s \in \mathbb{C}$ : $\left.\Re s=\sigma_{0}\right\}$ is everywhere dense in the set of the values of the series (2.1) on the set $\left\{z:\left|z_{1}\right|=\frac{1}{p_{1}^{\sigma_{0}}}, \ldots,\left|z_{m}\right|=\frac{1}{p_{m}^{\sigma_{0}}}\right\}$, [18]. We will be using this result in the particular case when the series (1.4) is just a Dirichlet polynomial. The same conclusion is easily deduced from a theorem of Kronecker, [13]. Denote by $\sigma_{u}$ the abscissa of uniform convergence of the series (1.4), that is, the infimum of those $\sigma$, so that the series (1.4) converges uniformly in the half-plane $\Pi_{\sigma}=\{s \in \mathbb{C}: \Re s>\sigma\}$. Let $\sigma_{b}$ be infimum of those $\sigma$ for which the series (1.4) is bounded (and naturally holomorphic) in the half-plane $\Pi_{\sigma}$. A non-trivial result of Bohr states that $\sigma_{u}=\sigma_{b},[17$, , 13]. Therefore, if $f(\Pi) \subset G$, where $G$ is a bounded domain in $\mathbb{C}$, then in every half-plane $\Pi_{\sigma}, \sigma>0$ the series (1.4) converges uniformly. Hence, for $\sigma$-fixed, we can find for every $\epsilon>0$ an index $k \in \mathbb{N}$ so that $\left|f(s)-P_{k}(s)\right|<\epsilon$, where the polynomial $P_{k}(s)$ is taken from (1.7). We remark that $P_{k}\left(\Pi_{\sigma}\right) \subset G_{\epsilon}$, where $G_{\epsilon}$ is an $\epsilon$-neighborhood of $G$. Therefore the polynomial

$$
\widetilde{P}_{k}(z)=\sum_{n=1}^{k} a_{n} z_{1}^{\alpha_{1}} \ldots z_{m}^{\alpha_{m}}
$$

in the corresponding polydisc $\mathcal{D}$ is such that $\widetilde{P}_{k}(\mathcal{D}) \subset G_{\epsilon}$. From Theorem 1.2 then follows that $R\left(\mathcal{D}, G_{\epsilon}\right) \geq R\left(\mathcal{D}, U_{1}\right)$ and therefore the claim

$$
\sum_{n=2}^{k}\left|a_{n}\right| \frac{1}{p_{1}^{\sigma}} \ldots \frac{1}{p_{m}^{\sigma}}<\operatorname{dist}\left(a_{1}, \partial \widetilde{G}_{\epsilon}\right)
$$

for $\sigma \leq \mathbf{b}\left(U_{1}\right)$ is valid. Taking the limit in (2.2) for $k \longrightarrow \infty$ (this means $\epsilon \longrightarrow 0$ ), we obtain that for such a $\sigma$ the relation (1.6) holds, but instead of the proper inequality $<$, the inequality $\leq$ is now possible. But if we had equality there, then by taking smaller $\sigma$ we would have had obtained the converse inequality, which is impossible. Therefore $\mathbf{b}(G) \leq \mathbf{b}\left(U_{1}\right)$.

Let us assume now that on the boundary $\partial G$ there exists at least one regular point of convexity. Denote it by $p_{0}$. Then there exists a disk $U \subset G$ so that $p_{0} \in \partial U \cap \partial G \cap \partial \widetilde{G}$. Remark that homothety and parallel translation for the disk $U_{1}$ (that is all $a_{n}$ are replaced by $r a_{n}$ and after this $r a_{1}$ is replaced by $r a_{1}+c$, and therefore the new disc $U$ is $\left.r U_{1}+c\right)$ do not alter the condition (1.6) for the disc. Hence $\mathbf{b}(U)=\mathbf{b}\left(U_{1}\right)$. From here, it follows that $\mathbf{b}(G) \geq \mathbf{b}\left(U_{1}\right)=\mathbf{b}(U)$ since the corresponding set of holomorphic functions, which are represented by Dirichlet series in $\Pi$ and are satisfying $f(\Pi) \subset G$, is larger than 
the set of functions satisfying $f(\Pi) \subset U$. Thus, in this case we obtain $\mathbf{b}(G)=\mathbf{b}\left(U_{1}\right) \cdot \diamond$.

Corollary 2.1. If $G$ is a bounded, convex domain in $\mathbb{C}$ then the isometric Bohr abscissa $\mathbf{b}(G)$ does not depend from the choice of the domain $G$.

We point out that in the proof of the Theorem 2.1, we used the boundedness of the domain $G$ only to be able to approximate the Dirichlet series by a Dirichlet polynomial, in order to use the later's uniform convergence in the corresponding half-plane and thus to be able to apply Bohr result about the everywhere density of its values. Instead, one could demand that the series (1.4) converges absolutely in the half plane $\Pi$. Thus, one is able to formulate the following statement, where instead of isometric Bohr abscissa $\mathbf{b}(G)$ one considers $\mathbf{b}_{a}(G)$, defined in the same manner as $\mathbf{b}(G)$, but only for absolutely converging Dirichlet series in the half plane $\Pi$.

Theorem 2.2. Let $G$ be a domain in $\mathbb{C}, \widetilde{G} \neq \mathbb{C}$. Then the isometric Bohr abscissa $\mathbf{b}_{a}(G)$ is not greater $\mathbf{b}\left(U_{1}\right)$. If $\partial G$ contain at least one regular point of convexity, then $\mathbf{b}_{a}(G)=\mathbf{b}\left(U_{1}\right)$.

Theorem 2.3. Let $G$ be a bounded convex domain in $\mathbb{C}$. Then the following Rogosinski abscissas are equal: $\mathbf{r}(G)=\mathbf{r}\left(U_{1}\right)$.

Proof: The proof repeats the steps of the proof of the Theorem 2.1, but instead of Theorem 1.2 one uses the Theorem 1.4 and the following remark: the Dirichlet polynomial (1.7) can be described via the inequality

$$
\alpha_{1} \log 2+\alpha_{2} \log 3+\cdots+\alpha_{m} \log m \leq \log k,
$$

where the prime numbers are all prime numbers that appear in the prime decomposition of the integers $2,3,4, \ldots, k$. Approximating the logarithms in (2.3) with fractions so that no new integers appear and the existing ones remain, one can obtain the lattice

$$
\alpha_{1} d+\alpha_{2} d_{2}+\cdots+\alpha_{m} d_{m} \leq d,
$$

instead of the lattice (2.3), where $d, d_{i} \in \mathbb{Q}, i=1, \ldots, m$. Crucial fact here is that the new lattice is described by the same $m$-tuples $\left(\alpha_{1}, \ldots, \alpha_{m}\right)$ as in (2.3). For the final step it is enough to obtain a common denominator for the rational numbers $d, d_{i}, i=1, \ldots, m$ and get the lattice $\mathcal{A}$ from the Theorem 1.4. $\diamond$

We remark that defining the Rogosinski abscissa $\mathbf{r}_{a}(G)$ in the same manner as $\mathbf{r}(G)$, but only for absolutely convergent Dirichlet series in the half-plane, one obtains 
Theorem 2.4. If the domain $G$ is convex then $\mathbf{r}_{a}(G)=\mathbf{r}\left(U_{1}\right)$.

It is a well know fact that the Rogosinski radius is not smaller than the Bohr radius for power series in one and several complex variables (that is, the Rogosinski condition is satisfied in a disc (ball) of larger radius than the Bohr condition). Therefore, one might expect that the Rogosinski abscissa is not greater that the isometric Bohr abscissa for ordinary Dirichlet series. This is the content of the next result.

Theorem 2.5. Let $G$ be a convex bounded domain in $\mathbb{C}$. Then $\mathbf{r}(G) \leq$ $\mathbf{b}(G)$. If the domain $G \neq \mathbb{C}$ is just convex, then $\mathbf{r}_{a}(G) \leq \mathbf{b}_{a}(G)$.

Proof: Actually, if the domain $G$ is bounded and $\sigma>\mathbf{b}(G)$, then

$$
\left|P_{k}(s)-a_{1}\right|=\left|\sum_{n=2}^{k} \frac{a_{n}}{n^{s}}\right| \leq \sum_{n=2}^{k}\left|\frac{a_{n}}{n^{s}}\right| \leq \sum_{n=2}^{\infty}\left|\frac{a_{n}}{n^{s}}\right|<\operatorname{dist}\left(a_{1}, \partial G\right) .
$$

But the obtained inequality

$$
\left|P_{k}(s)-a_{1}\right| \leq \operatorname{dist}\left(a_{1}, \partial G\right)
$$

means geometrically that $P_{k}\left(\Pi_{\sigma}\right) \subset G$. Thus, if $\sigma>\mathbf{b}(G)$, then $\sigma>$ $\mathbf{r}(G)$ also. Therefore $\mathbf{r}(G) \leq \mathbf{b}(G)$. The case when the boundedness of the convex domain $G$ is not required is considered analogously. $\nabla$.

Remark 2.1. We point out the following open problems:

1. Is it possible to remove the condition on the boundedness of the domain $G$ in the statements of the Theorems 2.1, 2.4 and the Corollary 2.2?

2) Can one prove the second parts of the Theorems 2.1, 2.3 without the assumption on the existence of at least one regular point of convexity?

\section{Estimates for the Bohr And Rogosinski ABScissas}

Theorem 3.1. Let $G$ be a convex bounded domain in $\mathbb{C}$. Then the isometric Bohr abscissa satisfies

$$
\mathbf{b}(G) \leq 1.7267 \text {. }
$$

If $G$ is a domain in $\mathbb{C}, \widetilde{G} \neq \mathbb{C}$, then the isometric Bohr abscissa $\mathbf{b}_{a}(G)$ satisfies the same estimate.

Proof: Denote by $\Omega(n)$ the number of the prime divisors (counted with their multiplicity ) of the natural number $n$. Pivotal for us is the following result ([12], Prop.2.1): let $f$ be like in (1.4) and $\|f\|=1$, then for $k \geq 1$ one has

$$
\left(\sum_{\Omega(n)=k, n \geq 2}\left|a_{n}\right|^{2}\right)^{\frac{1}{2}} \leq 1-\left|a_{1}\right|^{2}
$$


Furthermore

$$
\begin{aligned}
\sum_{k=1}^{\infty} \frac{\left|a_{k}\right|}{n^{\sigma}} & \leq\left|a_{1}\right|+\sum_{k=1}^{\infty}\left(\sum_{\substack{\Omega(n)=k, n \geq 2}}\left|a_{n}\right|^{2}\right)^{\frac{1}{2}}\left(\sum_{\substack{\Omega(n)=k, n \geq 2}} \frac{1}{n^{2 \sigma}}\right)^{\frac{1}{2}} \\
& \leq\left|a_{1}\right|+\left(1-\left|a_{1}\right|^{2}\right) \sum_{k=1}^{\infty}\left(\sum_{\substack{\Omega(n)=k, n \geq 2}} \frac{1}{n^{2 \sigma}}\right)^{\frac{1}{2}}
\end{aligned}
$$

Consider now the equation

$$
\sum_{k=1}^{\infty}\left(\sum_{\substack{\Omega(n)=k, n \geq 2}} \frac{1}{n^{2 \sigma}}\right)^{\frac{1}{2}}=\frac{1}{2} .
$$

If $\sigma_{0}$ is the unique solution of the equation (3.3), then

$$
\sum_{k=1}^{\infty} \frac{\left|a_{k}\right|}{n^{\sigma_{0}}} \leq\left|a_{1}\right|+\left(1-\left|a_{1}\right|^{2}\right) \frac{1}{2} \leq 1
$$

since $\left|a_{1}\right| \leq 1$. Therefore $\mathbf{b}\left(U_{1}\right) \leq \sigma_{0}$. The solution of the equation (3.3) was obtained numerically, using Maple. Thus the estimate $\sigma_{0}<1.7267$ was obtained. $\diamond$

Corollary 3.1. Let $G$ be a bounded domain in $\mathbb{C}$. Assume also that $\partial G$ contains at least one regular point of convexity. Then

$$
1.5850 \cdots=\frac{\log 3}{\log 2} \leq \mathbf{b}(G)<1.7267 .
$$

If one does not require the boundedness of the domain $G \neq \mathbb{C}$, then $\mathbf{b}_{a}(G)$ satisfies the same estimates.

Theorem 3.2. Let $G$ be a convex bounded domain in $\mathbb{C}$. Then, if the function $f$ is from (1.5) and $\|f\|=1$, then for every such $f$

$$
\left|a_{1}\right|^{2}+\sum_{k=2}^{\infty} \frac{\left|a_{k}\right|}{n^{\sigma}} \leq 1
$$

where $1 \leq \sigma<1.2061$.

Proof: From (3.2), we obtain as before, that the left hand-side in (3.4) is not larger than

$$
\left|a_{1}\right|^{2}+\left(1-\left|a_{1}\right|^{2}\right) \sum_{k=1}^{\infty}\left(\sum_{\substack{\Omega(n)=k, n \geq 2}} \frac{1}{n^{2 \sigma}}\right)^{\frac{1}{2}} .
$$


BOHR AND ROGOSINSKI ABSCISSAS FOR ORDINARY DIRICHLET SERIES. 9

Consider the equation

$$
\sum_{k=1}^{\infty}\left(\sum_{\substack{\Omega(n)=k, n \geq 2}} \frac{1}{n^{2 \sigma}}\right)^{\frac{1}{2}}=1 .
$$

If $\sigma_{0}$ is the root of the equation (3.5), then for $\sigma=\sigma_{0}$ the relation (3.4) holds. The equation (3.5) was solved numerically by using Maple to obtain the estimate $\sigma_{0}<1.2061$.

On the other hand, a particular case of the Dirichlet series (1.4) is the series

$$
f(s)=\sum_{n=0}^{\infty} \frac{a_{n}}{2^{n s}},
$$

which is a power series relatively to the variable $\frac{1}{2^{s}}$. It is known $([27])$ that the best value for the radius $r$, for which the relation

$$
\left|c_{0}\right|^{2}+\sum_{k=1}^{\infty}\left|c_{k}\right| r^{k} \leq 1
$$

is valid for every power series (1.1) satisfying $\left|f\left(z_{1}\right)\right|<1$ in the unit disk $U_{1}$, is $\frac{1}{2}$. Then for the Dirichlet series (3.6) we obtain

$$
\frac{1}{2^{\sigma_{0}}}=\frac{1}{2}
$$

that is $\sigma_{0}=1$. Since the series (3.6) is a particular case of the series (1.4), we deduce that $\sigma$ from Theorem 3.3 is greater or equal to $1 . \diamond$ Analogously one can prove the following

Theorem 3.3. Let $G$ be a convex bounded domain in $\mathbb{C}$. Then the Rogosinski abscissa $\mathbf{r}(G) \geq 1$. If the domain $G \neq \mathbb{C}$ is not bounded then the Rogosinski abscissa $\mathbf{r}_{a}(G)$ satisfies the same inequality.

Remark 3.1. We conclude the present paper by by stating the following hypothesis: for every convex bounded domain $G \subset \mathbb{C}$ the equality $\mathbf{r}(G)=1$ is true. If the domain $G \neq \mathbb{C}$ is convex, but not necessarily bounded then also $\mathbf{r}_{a}(G)=1$.

\section{REFERENCES}

[1] L.Aizenberg, Multidimensional analogues of Bohr's theorem on power series, Proc. Amer. Math. Soc. 128 (2000), 1147-1155.

[2] L.Aizenberg, Bohr Theorem, Encyclopedia of Mathematics, supplement II (ed. M.Hezewinkel, Kluwer, Dordrecht 2000), 76-78.

[3] L.Aizenberg, A generalization of Caratheodory inequality and the Bohr radius for multidimnesional power series, Operator Theory, Advances and Applications 158 (2005), 87-94. 
[4] L.Aizenberg, Generalization of results about the Bohr radius for power series, To appear in Studia Math.

[5] L.Aizenberg, A.Aytuna, P.Djakov, An abstract approach to Bohr's phenomenon, Proc. Amer. Math. Soc. 128 (2000), 2611-2619.

[6] L.Aizenberg, A.Aytuna, P.Djakov Generalization of Bohr's theorem for bases in spaces of holomorphic functions of several complex variables, J. of Math. Anal. Appl. 258 (2001), 428-447.

[7] L.Aizenberg, I.B.Grossman, Yu.F. Korobeinik, Some remarks on Bohr radius for power series, Isv. Vyssh. Ucheb.Zav. Mat. 2002, no.10, 3-10.

[8] L.Aizenberg, M.Elin, D.Shoikhet, On the Rogosinski radius for holomorphic mappings and some of its applications, Studia Math. 168(2)(2005), 147-158.

[9] L.Aizenberg, E.Liflyand, A.Vidras, Multidimensional analogue of the van der Corput-Visser inequality and its application to the estimation of the Bohr radius, Anal.Pol.Math. 80 (2003), 47-54.

[10] L.Aizenberg, N.Tarkhanov, A Bohr phenomenon for elliptic equations. Proc. London Math. Soc. (3) 82 (2001), no. 2, 385-401

[11] L.Aizenberg, A.Vidras, On the Bohr radius of two classes of holomorphic functions, Siberian Math. J. 45 (2004), no. 4, 606-617.

[12] R.Balasubramanian. B.Calado, H.Queffélecc, The Bohr inequality for ordinary Dirichlet series, Studia Math. 175 (2006), 285-304.

[13] F.Bayart, The Hardy spaces of Dirichlet series and their composition operators, Monatsh. Math. 136 (2002), 203-236.

[14] C.Bénéteau, A.Dalhner, D.Khavinson, Remarks on the Bohr phenomenon, Comput. Method. Funct. Theory 4 (2004), 1-19.

[15] H.P.Boas, Majorant series, J. Korean Math. Soc. 37 (2000), 321-337.

[16] H.P.Boas, D.Khavinson, Bohr's power series theorem in several variables, Proc. Amer. Math. Soc. 125 (1997), 2975-2979.

[17] H.Bohr, Über die gleichmässige Konvergenz Dirichletscher Reihen, J.Reine Angew. Math. 143 (1913), 203-211.

[18] H.Bohr, Über die Bedeutung der Potenzreichen unendlich vieler Variablen in der Theoria der Dirichletschen Reihen $\sum \frac{a_{n}}{n^{s}}$, Nach. Acad. Wiss. Göttingen (1913), 441-488.

[19] H.Bohr, A theorem conserning power series, Proc. London Math.Soc. 13 (1914), 1-5.

[20] A.Defant, L. Frerick, A logarithmic lower bound for multi-dimensional Bohr radii, Israel J. of Math. 152 (2006), 17-28.

[21] A.Defant, D.Garsia, and M.Maestre, Bohr's power series theorem and local Banach space theory, J.Reine uns Angew. Math. 557 (2003), 173-197.

[22] A.Defant, D.Garsia, and M.Maestre, Estimates for the first and second Bohr radii of Reinhardt domains, J.Approx. Theory 128 (2004), 53-68.

[23] S.Dineen, R.Timoney, Absolute bases, tensor products and a theorem of Bohr, Stud. Math. 94 (1989), 227-234.

[24] P.B.Djakov, M.S.Ramanujan, A remark on Bohr's theorem and its generalizations, J. Analysis, 8 (2000), 65-77.

[25] G.Kresin, V.Maz'ya, Sharp Bohr's type real part estimates, preprint, 2006, 1-16.

[26] E.Landau, D.Gaier, Darstellung und Begrundung einiger neuerer Ergebnisse der Funktionentheorie, Springer-Verlag, 1986. 
BOHR AND ROGOSINSKI ABSCISSAS FOR ORDINARY DIRICHLET SERIES11

[27] V.I.Paulsen, G.Popescu, D.Singh, On Bohr inequality, Proc. Lond. Math. Soc. 85 (2002), 493-515.

[28] W.Rogosinski, Über Bildschranken bei Potenzreihe und ihren Abschnitten, Math.Z. 17 (1923), 260-276.

[29] I.Schur und G.Szego, Über die Abschnitte einer im Einheitskreise Geschränkten Potenzreihe, Sitz.-Ber. Preuss. Acad. Wiss. Berlin Phys.-Math. Kl. (1925), 545560.

[30] S.Sidon, Über einen Satz von Herrn Bohr, Math. Zeit. 26 (1927), 731-732.

[31] M.Tomič, Sur une théorèm de H.Bohr, Nath. Scandin. 11 (1963), 103-106.

Department of Mathematics, Bar-Ilan University

RAMAT-Gan 52900, ISRAEL.

Department of Mathematics, Holon Institute of Technology, P.O.B 305, Holon 58102, IsRael.

Department of Mathematics and Statistics, Univ.of Cyprus, Nicosia 1678, Cyprus.

E-mail address: aizenbrg@macs.biu.ac.il, gotlib@hit.ac.il

E-mail address: msvidras@ucy.ac.cy 